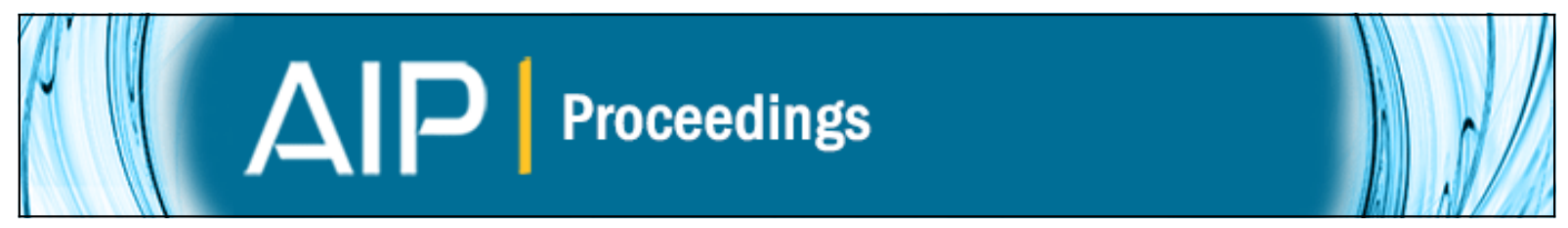

\title{
The Texas petawatt laser and current experiments
}

Mikael Martinez, Woosuk Bang, Gilliss Dyer, Xiaoming Wang, Erhard Gaul, Teddy Borger, Martin Ringuette, Michael Spinks, Hernan Quevedo, Aaron Bernstein, Michael Donovan, and Todd Ditmire

Citation: AIP Conference Proceedings 1507, 874 (2012); doi: 10.1063/1.4773814

View online: http://dx.doi.org/10.1063/1.4773814

View Table of Contents: http://scitation.aip.org/content/aip/proceeding/aipcp/1507?ver=pdfcov

Published by the AIP Publishing

\section{Articles you may be interested in}

A $5 \mu \mathrm{m}$ wavelength laser for dielectric laser acceleration

AIP Conf. Proc. 1507, 893 (2012); 10.1063/1.4773818

Calibration of the neutron detectors for the cluster fusion experiment on the Texas Petawatt Laser

Rev. Sci. Instrum. 83, 063504 (2012); 10.1063/1.4729121

Preparation For Laser Wakefield Experiments Driven by the Texas Petawatt Laser System

AIP Conf. Proc. 1086, 177 (2009); 10.1063/1.3080901

Progress on the MIT Photonic Band Gap Accelerator Experiment

AIP Conf. Proc. 737, 783 (2004); 10.1063/1.1842623

Acceleration of injected electrons in a laser wakefield experiment

Phys. Plasmas 6, 2903 (1999); 10.1063/1.873248 


\title{
The Texas Petawatt Laser and Current Experiments
}

\author{
Mikael Martinez ${ }^{\mathrm{a}}$, Woosuk Bang ${ }^{\mathrm{a}}$, Gilliss Dyer ${ }^{\mathrm{a}}$, Xiaoming Wang ${ }^{\mathrm{b}}$ Erhard Gaul $^{\mathrm{a}}$, \\ Teddy Borger ${ }^{\mathrm{a}}$, Martin Ringuette ${ }^{\mathrm{a}}$, Michael Spinks ${ }^{\mathrm{a}}$, Hernan Quevedo ${ }^{\mathrm{a}}$, Aaron \\ Bernstein $^{\mathrm{a}}$, Michael Donovan ${ }^{\mathrm{a}}$ and Todd Ditmire ${ }^{\mathrm{a}}$
}

${ }^{a}$ Center for High Energy Density Science, The University of Texas at Austin 1 University Station Austin, TX 78712

${ }^{b}$ Laser Plasma Acceleration Group, Department of Physics, The University of Texas at Austin 1 University Station Austin, TX 78712

\begin{abstract}
The Texas Petawatt Laser is operational with experimental campaigns executed in both F/40 and F3 target chambers. Recent improvements have resulted in intensities of $>2 \times 10^{21} \mathrm{~W} / \mathrm{cm}^{2}$ on target. Experimental highlights include, accelerated electron energies of $>2 \mathrm{GeV}$, DD fusion ion temperatures $>25 \mathrm{keV}$ and isochorically heated solids to $10-50 \mathrm{eV}$.

Keywords: OPCPA, Mixed Nd:glass, Petawatt, laser-cluster fusion, neutron source, laser wakefield acceleration, warm dense matter, and isochoric heating.

PACS: 42.2.Eq, 42.65.Rc, Ky, 42.70.Hj, Mp, 29.25.Dz, 52.50.Jm, and 25.45. -Z, 36.40.Gk 52.38.Kd, 52.38.Hb
\end{abstract}

\section{FACILITY DESCRIPTION}

The Texas Petawatt Laser Facility (TPW) is an internationally unique research tool located at the University of Texas at Austin's Center for High Energy Density Science. Research conducted in the facility is primarily centered on the field of High Energy Density Science (HED). The TPW operates year around and supports up to 10 experimental campaigns over 200 days and a total shot count of 1,200 shots. The experimental users are composed of UT Physics Faculty, DOE National Laboratories, US academic institutions and international collaborators. A formal User Program organizes the annual shot calendar. The TPW is designed to deliver a wide range of experimental parameters on target. To accomplish this, the User can choose between either a high intensity $\left(>10^{21}\right.$ $\mathrm{W} / \mathrm{cm}^{2}$ ) $\mathrm{f} / 3$ target chamber or a long focus $\mathrm{f} / 40$ geometry. The laser is capable of delivering $180 \mathrm{~J}$ pulses in $170 \mathrm{fs}$ at a center wavelength of $1057 \mathrm{~nm}$. See Figure 1 below for a facility layout.

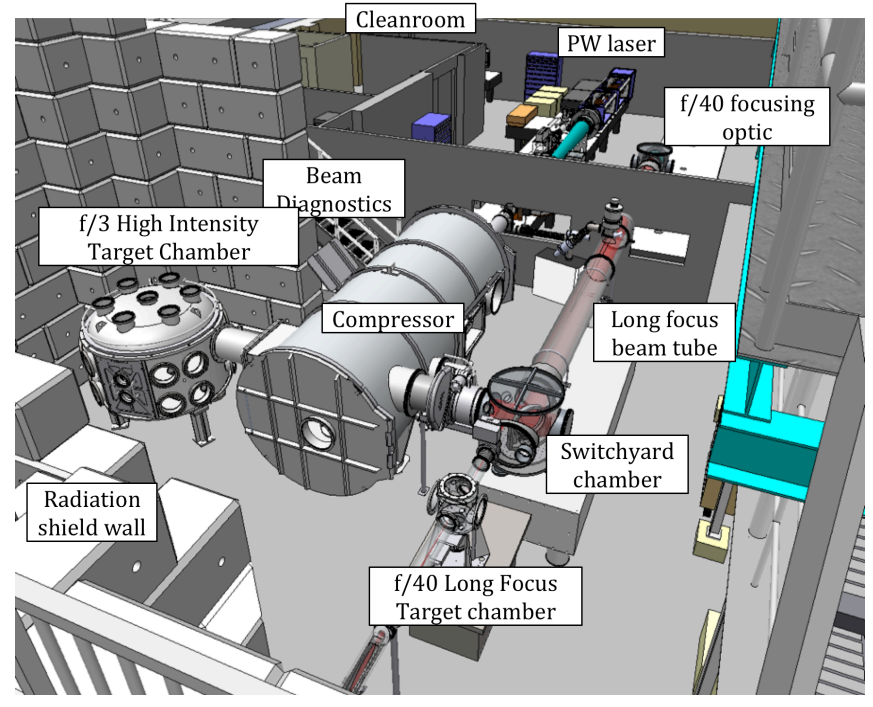

FIGURE 1. The Texas Petawatt Laser Facility provides two experimental chambers to support a wide range of experimental missions. 


\section{LASER SYSTEM ARCHITECTURE}

At $180 \mathrm{~J}, 170 \mathrm{fs}$ and 1 shot/hour, The Texas Petawatt Laser is unique. Typically, petawatt class lasers operate at $30 \mathrm{~J}, 30 \mathrm{fs}, 500 \mathrm{~J}, 500 \mathrm{fs}$ or $1 \mathrm{~kJ}, 1 \mathrm{ps}[1,2,3]$. Currently, however, a whole new class of 10 PW systems are funded and in the design phase [4]. The Texas Petawatt leverages a novel, hybrid approach of Optical Parametric Chirped Pulse Amplification (OPCPA) and mixed Nd:glass amplification [5]. This architecture takes advantage of the high gain $\left(>10^{10}\right)$, broad bandwidth $(>30 \mathrm{~nm})$ OPCPA as well as the large aperture $(>24 \mathrm{~cm})$, high-energy storage, high optical quality characteristics of Nd:glass. Additionally, keeping the net gain in Nd:glass ( 400) and using two compositions of $\mathrm{Nd}$ :glass (silicate and phosphate), this design mitigates the unwanted effects of gain narrowing. This hybrid approach enables the Texas Petawatt to delivery energies and pulse durations relevant for HED science missions. Figure 2, shown below describes the architecture of the laser system, design parameters of subsystems as well as emission spectra overlap of the mixed $\mathrm{Nd}$ :silicate and $\mathrm{Nd}$ :phosphate.

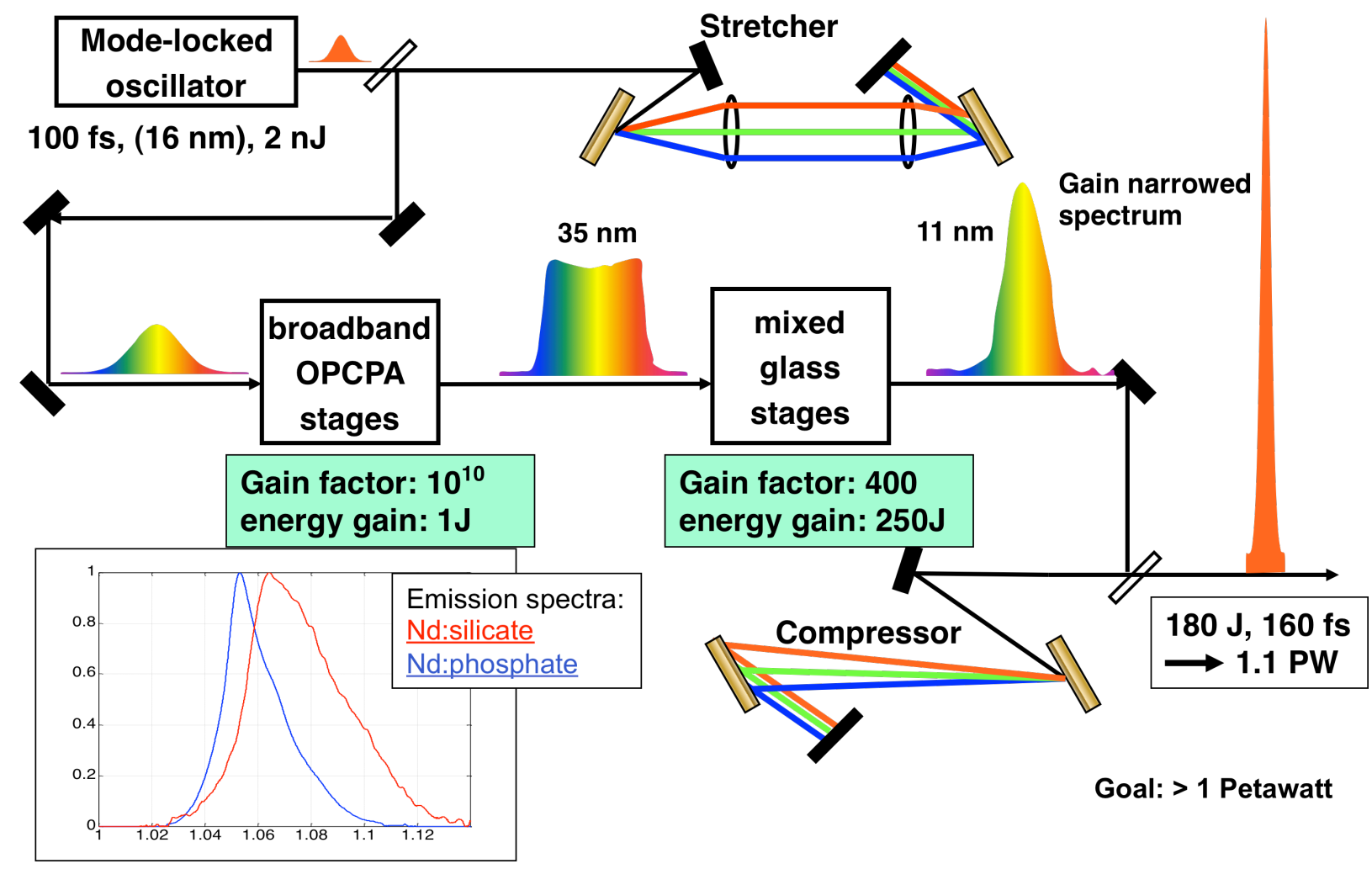

FIGURE 2. The system architecture includes OPCPA, mixed Nd:glass with design performance targets as well as emission spectra of the two $\mathrm{Nd}$ :glass compositions used to achieve the bandwidth required to $100 \mathrm{fs}$ pulses.

\section{RECENT LASER SYSTEM PERFORMANCE}

Recently, the Texas Petawatt has demonstrated excellent system performance on a number of experimental campaigns. Due to laser damage on the post compression, near normal incidence mirrors, the system has been derated to $100 \mathrm{~J}$. Despite the de-rated energy, the system still delivers greater that $1 \times 10^{21} \mathrm{~W} / \mathrm{cm}^{2}$ on $30 \%$ or more of the shots in the $\mathrm{f} / 3$ target chamber. In the $\mathrm{f} / 40$ long focus chamber, the system is delivers $28 \%$ of the total energy into a $160 \mu \mathrm{m}$ spot. Figure 3 shows a shot report that represents the average performance of a shot into the $\mathrm{f} / 3$ chamber. 


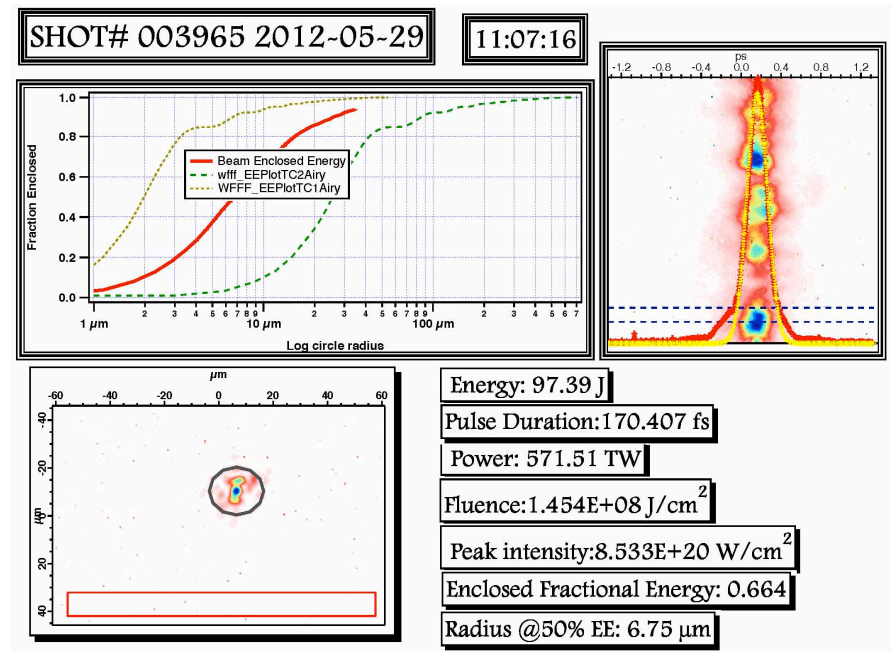

(a)

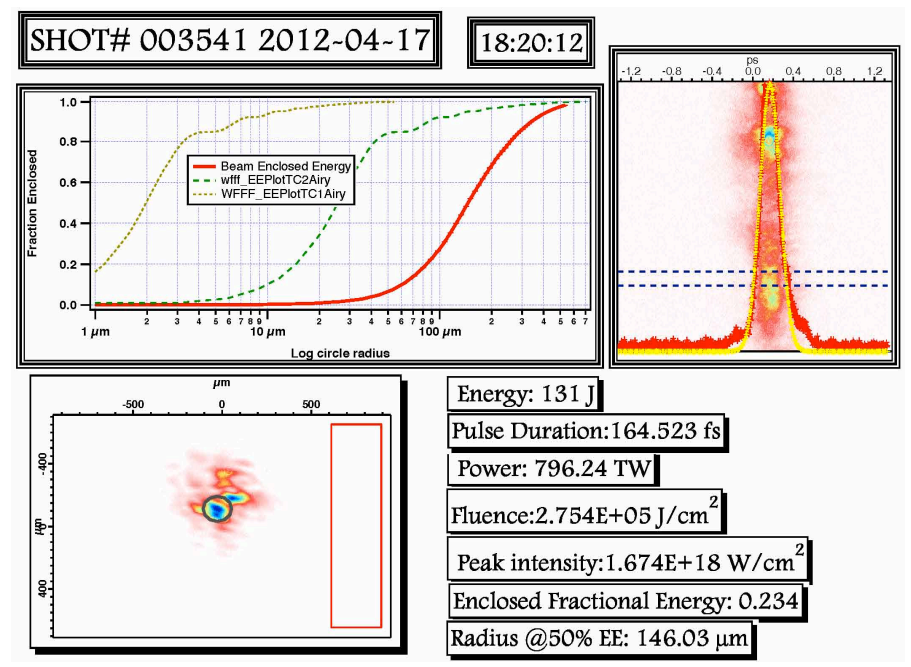

(b)

FIGURE 3. (a) Shot report representing average laser performance for shots into the $f / 3$ chamber. (b) Shot report representing average laser performance for shots into the $\mathrm{f} / 40$ chamber.

TABLE a). This table shows relevant laser parameters taken for 100 shots in the $f / 3$ chamber and 18 shots in the $\mathrm{f} / 40$ chamber. Each parameter is measured on every shot.

\begin{tabular}{lcc}
\hline Laser Parameter & $\mathbf{f} / \mathbf{3}$ Chamber & $\mathbf{f} / \mathbf{4 0}$ Chamber \\
\hline Average Energy & $97.6 \mathrm{~J}$ & $132 \mathrm{~J}$ \\
Std. Dev & $7.7 \mathrm{~J}$ & $12 \mathrm{~J}$ \\
Avg. Pulse Duration & $167 \mathrm{fs}$ & $178 \mathrm{fs}$ \\
Avg. Enclosed Energy & $65 \%(10 \mu \mathrm{m}$ dia. $)$ & $28 \%(160 \mu \mathrm{m}$ dia. $)$ \\
Peak Intensity & $1.04 \times 10^{20} \mathrm{~W} / \mathrm{cm}^{2}$ & $1.04 \times 10^{18} \mathrm{~W} / \mathrm{cm}^{2}$ \\
& $\left(\sigma=3.5 \times 10^{20} \mathrm{~W} / \mathrm{cm}^{2}\right)$ & $\left(\sigma=3.5 \times 10^{20} \mathrm{~W} / \mathrm{cm}^{2}\right)$ \\
\hline
\end{tabular}




\section{RECENT EXPERIMENTAL PROGRESS}

The Texas Petawatt facility supports approximately 10 experimental campaigns per year. Each campaign can run from 3-5 weeks with laser maintenance scheduled between runs. Users can submit proposals for consideration to be scheduled. The shot calendar looks 6-12 months out. Below are highlights from 3 experiments in 2011-2012.

\section{Laser Wakefield Acceleration}

One series of campaigns produced laser wakefield acceleration of quasi monoenergetic electrons to $2.4 \mathrm{GeV}$ in a low electron density $\left(\mathrm{n}_{\mathrm{e}}=3.3 \times 10^{17} \mathrm{~cm}^{-3}\right)$ petawatt driven wakefield and realized electron self-injection at $\mathrm{n}_{\mathrm{e}}=$ $1.0 \times 10^{17} \mathrm{~cm}^{-3}[6]$. The self-injected electrons had a low divergence angle less than $0.5 \mathrm{mrad}$ FWHM on individual shots and a pointing stability less than $2.5 \mathrm{mrad}$. was observed over multiple shots. The electron charge we observed in experiments varied from several tens PC to hundreds PC, which strongly depend on the plasma density and laser power. These experimental results make the single stage, self-injected, multi-GeV electron acceleration with PW lasers at low plasma density $\left(\mathrm{n}_{\mathrm{e}} \sim 10^{17} \mathrm{~cm}^{-3}\right)$ very promising.
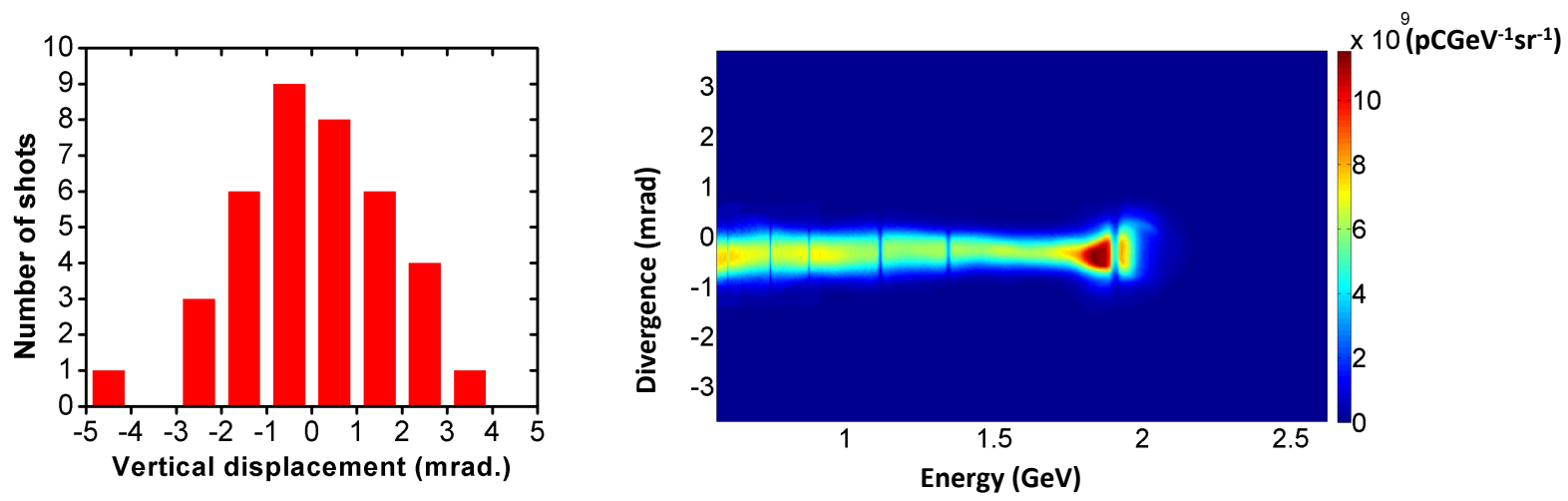

FIGURE 4. (a) Statistical results of the electron beam vertical displacement over multiple system laser shots; (b) Quasi mono energegtic spectrum reaching $2 \mathrm{GeV}$.

\section{Cluster Fusion}

In another experimental campaign, we studied interactions of petawatt pulses with two different types of cluster targets: one a mixture of deuterium clusters and ${ }^{3} \mathrm{He}$ gas, and one of deuterated methane clusters and ${ }^{3} \mathrm{He}$ [7]. These interactions produced deuterium ions energetic enough to initiate D- ${ }^{3} \mathrm{He}$ nuclear fusion reactions as well as the DD fusion reactions inside the fusion plasma. By comparing the neutron yield from $\mathrm{D}(\mathrm{D}, \mathrm{n})^{3} \mathrm{He}$ reactions with the proton yield from $\mathrm{D}\left({ }^{3} \mathrm{He}, \mathrm{p}\right){ }^{4} \mathrm{He}$ reactions, we successfully measured the ion temperature of fusion plasmas at the time of the fusion reactions. The measurements of ion temperatures with fusion yields were consistent with independently measured ion temperatures from the ion time-of-flight measurements, and the measured temperature of deuterium ions was as high as $25 \mathrm{keV}$. The following figure illustrates the schematic setup for the mixture cluster fusion experiments along with the relevant fusion reactions. 


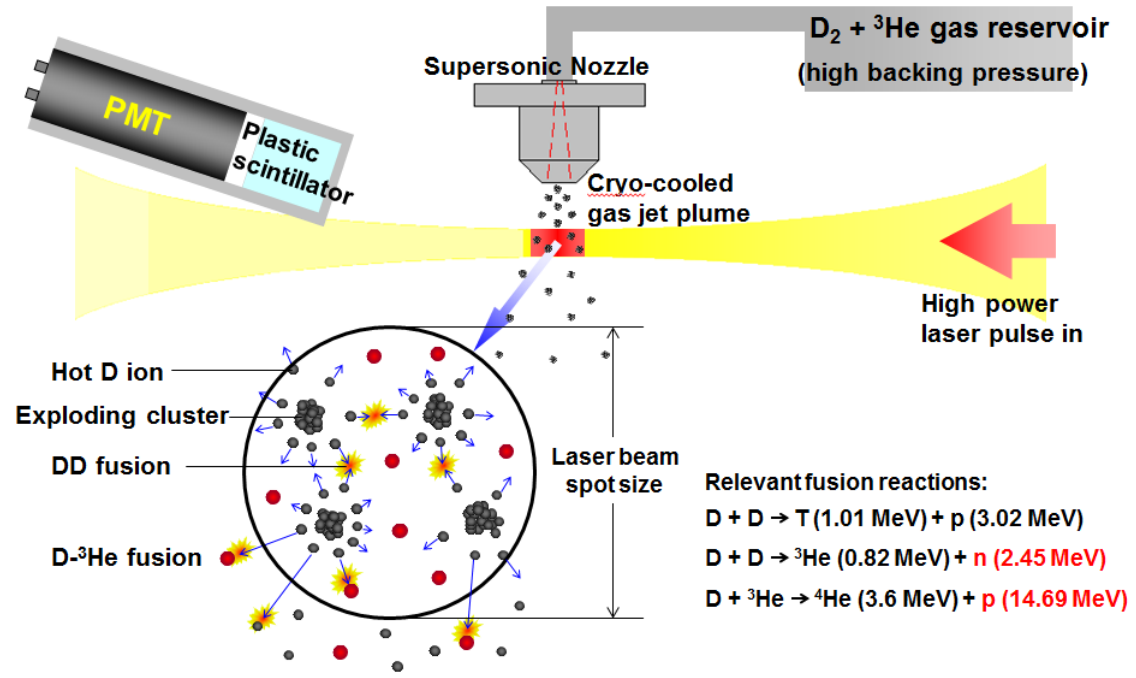

FIGURE 4. Schematic diagram illustrating the laser-cluster interaction.

\section{Warm Dense Matter}

Building on earlier proof of principle experiments on other laser systems [8,9] we have begun a campaign to study the equation of state (EOS) properties of a number of materials of interest in the temperature and density regime known as warm dense matter (WDM). Using a well-characterized, intense proton beam generated by petawatt laser-solid interaction with a source foil, a secondary sample foil is rapidly heated to temperatures of 1 to $100 \mathrm{eV}$. The heating is isochoric and ultrafast measurement techniques are used to discern the expansion and temperature of the heated sample. Comparing the expansion rate to the thermal emission and using the measured proton spectrum to estimate the energy density, we produce EOS data for conditions that have scarcely been measured and for which theoretical models are difficult to achieve. We have worked with the EOS group at LLNL to determine materials of interest, leading the initial experiments to focus on transition metals. So far we have made several complete measurements on $\mathrm{Cu}$ and $\mathrm{Ag}$ WDM states with temperatures of up to $65 \mathrm{eV}$ [10].

\section{REFERENCES}

1. J. Sung, S. Lee et al., 0.1 Hz 1.0 PW Ti:sapphire Laser, Optics Letters 35, 3021-3023 (2010).

1. J. Schwartz, P. Rambo, M. Geissel et al., Journal of Physics: Conference Series 85, 032020-24 (2008).

2. L. Waxer, M. Guardalben et al., "The OMEGA EP High-Energy, Short-Pulse Laser System" in High-Energy Short-Pulse Lasers and Technology (JThB)-2008, Conference on Lasers and Electro-Optics Technologies San Jose, California.

4. J.P. Chambaret, P. Georges et al., "The Extreme Light Infrastructure Project ELI and Its Prototype APOLLON/ILE: "The Associated Laser Bottlenecks" in High Peak Power Laser Technology I (FMI)-2009, Frontiers in Optics (FiO) San Jose, California.

5. E. Gaul, M. Martinez, J. Blakeney et al., Applied Optics, 49, 9, p1676 ff, (2010).

6. X. Wang, R. Zgadzai, S. A. Yi et al., Phy. of Plasmas, accepted. (2012).

7. W. Bang, G. Dyer, H. Quevedo et al., Phys. Rev. Lett., in preparation. (2012).

8. G. M. Dyer, A. C. Bernstein, et al., Phys. Rev. Lett. 101, 15002 (2008).

9. A. Mancic, J. Robiche, P. Antici, P. Audebert, C. Blancard, P. Combis, F. Dorchies, G. Faussurier, S. Fourmaux, M. Harmand,

R. Kodama, L. Lancia, et al., High Energy Density Physics 6, 21 (2010).

10. G.M. Dyer in preparation 